REPRESENTATION THEORY

An Electronic Journal of the American Mathematical Society

Volume 3, Pages 90-104 (June 22, 1999)

S $1088-4165(99) 00074-6$

\title{
CONSTRUCTION OF ARBITRARY KAZHDAN-LUSZTIG POLYNOMIALS IN SYMMETRIC GROUPS
}

\author{
PATRICK POLO
}

\begin{abstract}
To each polynomial $P$ with integral nonnegative coefficients and constant term equal to 1 , of degree $d$, we associate a certain pair of elements $(y, w)$ in the symmetric group $S_{n}$, where $n=1+d+P(1)$, such that the Kazhdan-Lusztig polynomial $P_{y, w}$ equals $P$. This pair satisfies $\ell(w)-\ell(y)=$ $2 d+P(1)-1$, where $\ell(w)$ denotes the number of inversions of $w$.
\end{abstract}

\section{INTRODUCTION}

For every Coxeter group $(W, S)$, Kazhdan and Lusztig defined in [11] a polynomial $P_{y, w}(q) \in \mathbb{Z}[q]$ for each pair $(y, w)$ of elements of $W$ such that $y \leq w$ in the Bruhat-Chevalley order. It has constant term equal to 1 and, when $y \neq w$, degree at most equal to $(\ell(w)-\ell(y)-1) / 2$, where $\ell$ denotes the length function on $(W, S)$. When $(W, S)$ is a finite or affine Weyl group, they showed in [12] that the $P_{y, w}(q)$ have nonnegative coefficients by relating them to the intersection cohomology of Schubert varieties.

In spite of their rather elementary definition in [11], the polynomials $P_{y, w}$ are quite difficult to compute explicitly. In view of the interpretation in terms of intersection cohomology of Schubert varieties, it seems that this difficulty reflects the rather complicated nature of the singularities of Schubert varieties in general. In fact, the only families of Kazhdan-Lusztig polynomials known so far correspond to situations where the geometry is simpler, namely Schubert varieties in Grassmannians $([13,17])$ and other minuscule $G / Q([3])$, or the incidence variety in $\mathbb{P}^{n} \times\left(\mathbb{P}^{n}\right)^{*}$ $([4])$.

In this paper we adopt the reverse approach and show that any polynomial $P \in 1+q \mathbb{N}[q]$ is a Kazhdan-Lusztig polynomial. Namely, we prove the following. Let $d=\operatorname{deg}(P)$ and $n=1+d+P(1)$. We may and shall assume that $d \geq 1$.

Theorem. There exists a pair of elements $y_{P}<w_{P}$ in the symmetric group $S_{n}$, explicitly defined, whose associated Kazhdan-Lusztig polynomial is P. Further, this pair satisfies $\ell\left(w_{P}\right)-\ell\left(y_{P}\right)=2 d+P(1)-1$.

Remarks. 1) In this construction, $P$ is of degree $\left(\ell\left(w_{P}\right)-\ell\left(y_{P}\right)-1\right) / 2$ if and only if $P(1)=2$, that is, if and only if $P$ equals $1+q^{d}$.

2) The special case where $P=1+q^{d}$ was known already: a simple geometric proof is given in $[4,4.6]$. Also, A. Lascoux has informed me that he had obtained, jointly with M.P. Schützenberger, a combinatorial proof in that case.

Received by the editors December 11, 1998 and, in revised form, April 30, 1999.

1991 Mathematics Subject Classification. Primary 14M15; Secondary 20F55, 20 G15.

(C)1999 American Mathematical Society 
The idea that this result should be true, as well as the precise conjectural form of the elements $y_{P}, w_{P}$, came from computations made in the symmetric group $S_{8}$ by using the computer program "Coxeter" written by Fokko Du Cloux ([7]). In order to prove that the postulated pair $(y, w)$ actually has $P$ as the Kazhdan-Lusztig polynomial, we use the interpretation in terms of intersection cohomology. Without loss, we shall assume that the ground field is $\mathbb{C}$, the field of complex numbers. Let $G=S L_{n}(\mathbb{C})$, let $B$ be a Borel subgroup and $X_{w} \subset G / B$ the Schubert variety corresponding to $w$. We consider a certain resolution of singularities $\pi: Z_{w} \rightarrow X_{w}$ (of Bott-Samelson type), similar to the ones considered by Zelevinsky ([17]). At the difference of the situation in [loc. cit.], here $\pi$ need not be a small map, nor even semi-small. Yet, thanks to the particular form of the permutations $w$ and $y$, we are able to decompose explicitly the restriction to the open subset $\bigcup_{y \leq z \leq w} B z B / B$ of $R \pi_{*}(\mathbb{C})$ as a direct sum of shifted Deligne-Goresky-MacPherson complexes, using the decomposition theorem ([2]), ideas of Deodhar $([6])$, and a monotonicity result due to Irving ([9]).

After introducing some notation in Section 1, we prove the theorem in Sections 2 and 3. More precisely, in Section 2 we associate to every nonconstant polynomial $P \in 1+q \mathbb{N}[q]$ a certain pair $y_{P}<w_{P}$ and show that the proof of the equality $P_{y_{P}, w_{P}}=P$ can be reduced to the case where $P-1$ is a monomial. We complete the proof in this case in Section 3. Finally, in Section 4 we establish, for any connected semi-simple group, an isomorphism theorem concerning certain locally closed subvarieties of Schubert varieties, which seems to have been overlooked in the literature, and which provides a geometric explanation (in the case of finite Weyl groups) for an equality of Kazhdan-Lusztig polynomials used in the reduction step in Section 2.

\section{Notation AND RECOLLECTIONS}

1.1. In Sections $1-3$, the ground field is $\mathbb{C}$, the field of complex numbers, and algebraic varieties are equipped with the complex topology. For such a variety $X$, we denote by $I C(X)$ the Deligne-Goresky-MacPherson complex, and by $\mathcal{I} \mathcal{H}^{i}(X)$ its (intersection) cohomology sheaves (see $[2,8,12,16]$ ).

1.2. The Ehresmann-Bruhat-Chevalley order. Let $S_{n}$ denote the symmetric group in $n$ letters. Let us first recall that every permutation $w \in S_{n}$ is determined by its code $c(w)$, which is the $n$-tuple $\left(c_{1}(w), \ldots, c_{n}(w)\right)$, where $c_{i}(w)$ equals the number of $j>i$ such that $w(j)<w(i)$. Then $\ell(w)$, the number of inversions of $w$, equals $\sum_{i} c_{i}(w)$.

Let us also recall that the Ehresmann-Bruhat-Chevalley order on $S_{n}$, denoted by $\leq$, can be described in terms of keys of permutations. The $r$-th key of $w$, denoted by $K_{r}(w)$, is the array obtained by arranging the elements of $w([1, r])$ in increasing order: $i_{1}<\cdots<i_{r}$. The set of $r$-keys is endowed with the product order; that is, $\left(j_{1}<\cdots<j_{r}\right) \leq\left(i_{1}<\cdots<i_{r}\right)$ if and only if $j_{s} \leq i_{s}$ for all $s$. It is well known that $y \leq w$ if and only if $K_{r}(y) \leq K_{r}(w)$ for all $r$. One deduces, in particular, that if $c_{i}(w) \geq c_{i}(y)$ for all $i$, then $w \geq y$.

Most often, we shall denote a permutation $w \in S_{n}$ by the sequence $\left(i_{1}, \ldots, i_{n}\right)$, where $i_{r}=w(r)$. Yet, the transposition which exchanges $i$ and $j$ is denoted, as usual, by $(i, j)$. Further, the transposition $(i, i+1)$ will sometimes be denoted by $s_{i}$. 
For future reference, let us also record here the following. Given $w \in S_{n}$ and $i<j$, it is well known and easy to check that $w(i, j)<w$ if and only if $w(i)>w(j)$, and in this case one has

$$
\ell(w(i, j))=\ell(w)-1-2 \#\{k \in] i, j[\mid w(i)>w(k)>w(j)\} .
$$

For $y, w \in S_{n}$, we shall write $w \rightarrow y$ to mean that $w>y$ and $\ell(w)=\ell(y)+1$.

1.3. Schubert varieties. For any $n \geq 2$, let $\mathcal{F}(n)$ denote the variety of complete flags $V^{1} \subset \cdots \subset V^{n-1} \subset \mathbb{C}^{n}$. Let $\left\{e_{1}, \ldots, e_{n}\right\}$ be the standard basis of $\mathbb{C}^{n}$, and for every $i$ let $\mathbb{C}^{i}$ denote the span of $e_{1}, \ldots, e_{i}$. The stabilizer of the standard flag $\mathbb{C}^{1} \subset \cdots \subset \mathbb{C}^{n-1}$ is the subgroup of upper triangular matrices, denoted by $B$, and $\mathcal{F}(n)$ identifies with $S L_{n}(\mathbb{C}) / B$. For every $w \in S_{n}$, let $\mathbf{V}_{w}$ denote the flag whose $i$-th term is the $\mathbb{C}$-span of $e_{w(1)}, \ldots, e_{w(i)}$. Then the Schubert variety $X_{w}$ is the closure (taken in the Zariski or, equivalently, complex topology) of the orbit $B \mathbf{V}_{w}$. It is well known that one has

$$
X_{w}=\left\{V^{1} \subset \cdots \subset V^{n-1} \subset \mathbb{C}^{n} \mid \operatorname{dim}\left(V^{p} \cap \mathbb{C}^{q}\right) \geq \#\{i \leq p \mid w(i) \leq q\}\right\} .
$$

1.4. The monomial case. The simpler case where $P-1$ is a monomial $a q^{d}$ will play an important role in the sequel. In fact, the proof in the general case reduces, at a crucial step, to this case (see 2.5-2.7).

For the convenience of the reader, let us describe explicitly here the pair $w>y$ associated to $1+a q^{d}$. Let $n=d+a+2$ and consider the following elements of $S_{n}$ :

$$
\begin{aligned}
& w=(d+2, d+3, \ldots, n, d+1, \ldots, 3,1,2), \\
& y=(1, d+2, \ldots, n-1, d+1, \ldots, 3,2, n) .
\end{aligned}
$$

Their codes are the $n$-tuples: $c(w)=\left((d+1)^{a+1}, d, d-1, \ldots, 2,0,0\right)$ and $c(y)=$ $\left(0, d^{a}, d-1, \ldots, 1,0,0\right)$, where $m^{b}$ denotes a sequence of $b$ terms equal to $m$. Thus $c_{i}(w) \geq c_{i}(y)$, for all $i$, and one has, therefore, $y<w$ and $\ell(w)-\ell(y)=2 d+a$. (Note that $\ell(w)-\ell(y)-1=2 d$ if and only if $a=1$ ).

We will prove in Section 3 that $P_{y, w}=1+a q^{d}$. First, we begin with some results in the general case.

\section{Majoration of $P_{y, w}$ And Reduction to the monomial Case}

2.1. Let $\left(a_{1}, \ldots, a_{d}\right)$ be a $d$-tuple of nonnegative integers, with $a_{d} \neq 0$, and let $P=1+a_{1} q+\cdots+a_{d} q^{d}$. Let $|a|=\sum_{i} a_{i}$ and $n=d+P(1)+1=d+|a|+2$. For $i=1, \ldots,|a|$, let $d_{i}$ be the smallest integer $s$ such that $i \leq a_{1}+\cdots+a_{s}$. (In other words, the $d_{i}$ 's are defined by the equality $\sum_{i=1}^{|a|} q^{d_{i}}=\sum_{s=1}^{d} a_{s} q^{s}$.) Note that $d_{i} \leq d_{i+1}$ for all $i$.

Consider now the elements $w$ and $y$ in $S_{n}$ defined by the following conditions: $w(i)=i+d_{i}+1$ for $i \leq|a|+1, w(n)=2$, and $w(i)>w(i+1)$ for $|a|+2 \leq i \leq n-2$, while $y(1)=1, y(i)=w(i-1)$ for $2 \leq i \leq|a|+1, y(i)=w(i)$ for $|a|+2 \leq i \leq$ $n-2, y(n-1)=2$ and $y(n)=n$. (More explicitly, one has $w(n-1)=1$ and $w(n-s)=a_{1}+\cdots+a_{s-1}+s+1$, for $s=2, \ldots, d$.)

Therefore, the codes of $w$ and $y$ are the following $n$-tuples: (here, $m^{a}$ denotes a sequence of $a$ terms equal to $m$; by convention this is the empty sequence if $a=0$ )

$$
\begin{aligned}
c(w) & =\left(2^{a_{1}}, \ldots,(d+1)^{a_{d}}, d+1, d, \ldots \ldots \ldots \ldots, 2,0,0\right), \\
c(y) & =\left(0,1^{a_{1}}, \ldots \ldots \ldots \ldots, d^{a_{d}}, d-1, d-2, \ldots, 1,0,0\right) .
\end{aligned}
$$


Thus, $c_{i}(w) \geq c_{i}(y)$ for all $i$. It follows that $w>y$ and that $\ell(w)-\ell(y)=|a|+2 d$. The main result of this paper is the following.

Theorem. One has $P_{y, w}=1+a_{1} q+\cdots+a_{d} q^{d}$.

The proof will be given in a number of steps.

2.2. First, one observes that the Schubert variety $X_{w}$ is the closed subvariety of $\mathcal{F}(n)$ consisting of those flags $V^{1} \subset \cdots \subset V^{n-1} \subset \mathbb{C}^{n}$ such that $V^{i} \subset \mathbb{C}^{i+d_{i}+1}$, for $i=1, \ldots,|a|$, and $\mathbb{C}^{1} \subset V^{n-1}$.

The union of the orbits $B \mathbf{V}_{z}$, for $z \in[y, w]$, is an open subset of $X_{w}$, that we shall denote by $C_{[y, w]}$. On the other hand, let $Z_{w}$ be the variety consisting of pairs of flags $V^{1} \subset \cdots \subset V^{n-1}$ and $U^{2} \subset \cdots \subset U^{|a|+1}$ in $\mathbb{C}^{n}$ which satisfy the relations of inclusion indicated in the diagram below:

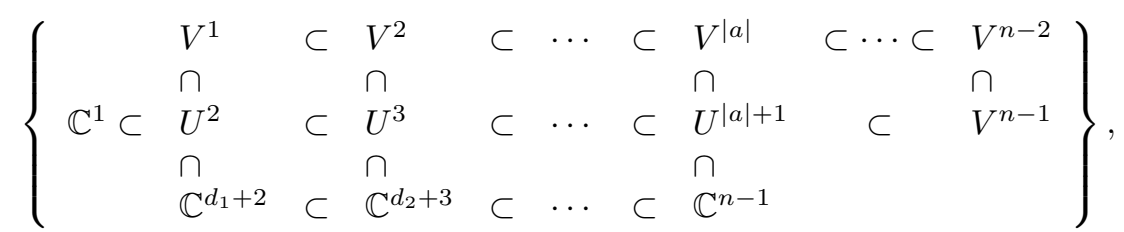

and let $\pi$ denote the natural projection $Z_{w} \rightarrow X_{w}$.

It is easily seen that $Z_{w}$ is smooth, since it is obtained as an iteration of locally trivial fibrations with fibres isomorphic to projective spaces. Moreover, $\pi$ is a resolution of singularities of $X_{w}$. In fact, $\pi$ is proper, and one has the following lemma. Let $X_{w}^{1}$ denote the closed, $B$-stable, subvariety of $X_{w}$ defined by the condition that $\mathbb{C}^{1} \subseteq V^{|a|}$.

Lemma. $\pi$ is an isomorphism over the complement of $X_{w}^{1}$.

Proof. Set $\Omega=X_{w} \backslash X_{w}^{1}$. Observe that if $\pi\left(V^{\bullet}, U^{\bullet}\right) \in \Omega$, then $U^{i+1}=V^{i}+\mathbb{C}^{1}$ for all $i \leq|a|$. Thus, $\pi$ is bijective over $\Omega$. Since $\pi$ is proper and $X_{w}$ normal $([15,14,1])$, it follows from Zariski's main theorem that $\pi$ is an isomorphism over $\Omega$.

We will see later that $\pi$ is not a small resolution if $d \geq 2$, and is not semi-small if $d \geq 3$ (see 3.2). Yet, using the decomposition theorem ([2]), plus ideas of Deodhar ([6]) and a result of Irving ([9]), we will show that, thanks to the particular form of $w$ and $y$, one can obtain enough information to decompose explicitly $j^{*} R \pi_{*}(\mathbb{C})$ as a direct sum of shifted Deligne-Goresky-MacPherson complexes, where $j$ denotes the inclusion $C_{[y, w]} \hookrightarrow X_{w}$.

2.3. Set $q=t^{2}$ and $H_{z, \pi}(t)=\sum_{i \geq 0} \operatorname{dim}\left(R^{i} \pi_{*} \mathbb{C}\right)_{\mathbf{V}_{z}} t^{i}$, for any $z \leq w$. On the one hand, since $\pi$ is proper, one has $\left(R^{i} \pi_{*} \mathbb{C}\right)_{\mathbf{V}_{z}} \cong H^{i}\left(\pi^{-1}\left(\mathbf{V}_{z}\right), \mathbb{C}\right.$ ) (see e.g. [10, Th.III.6.2]). On the other hand, since $\pi$ is $B$-equivariant and the $B$-orbits in $X_{w}$ are simply connected, the decomposition theorem ([2]) implies that

$$
R \pi_{*}(\mathbb{C}[\ell(w)]) \cong I C\left(X_{w}\right) \oplus \bigoplus_{v<w} E_{v} \otimes I C\left(X_{v}\right),
$$

where the $E_{v}$ are finite dimensional graded $\mathbb{C}$-vector spaces with the property that $\operatorname{dim} E_{v}^{i}=\operatorname{dim} E_{v}^{-i}$, for all $i$. Setting $E_{v}(t)=\sum_{i}\left(\operatorname{dim} E_{v}^{i}\right) t^{i}$, this is equivalent to 
the fact that $E_{v}\left(t^{-1}\right)=E_{v}(t)$. Moreover, by $[12,16]$, one has, for all $z \leq w$,

$$
\sum_{i} \operatorname{dim} \mathcal{I} \mathcal{H}^{i}\left(X_{w}\right)_{\mathbf{V}_{z}} t^{i}=t^{-\ell(w)} P_{z, w}(q) .
$$

One deduces that, for all $z \leq w$,

$$
H_{z, \pi}(t)=P_{z, w}(q)+\sum_{z \leq v<w} t^{\ell(w)-\ell(v)} E_{v}(t) P_{z, v}(q) .
$$

2.4. For all $i \leq|a|$, let $\gamma_{i}$ be the $\left(d_{i}+1\right)$-cycle which transforms $\left(i, n-d_{i}, \ldots, n-1\right)$ into $(n-1, i, \ldots, n-2)$ and let $v_{i}=w \gamma_{i}$.

Proposition. a) The $X_{v_{i}}$, for $i=1, \ldots,|a|$, are exactly the irreducible components of $X_{w}^{1}$. Further, one has $v_{i} \geq y$ and $\ell\left(v_{i}\right)=\ell(w)-d_{i}$ for all $i$.

b) For each $i$, the fibre $\pi^{-1}\left(\mathbf{V}_{v_{i}}\right)$ is isomorphic to projective space $\mathbb{P}^{d_{i}-1}$ and, therefore, $t^{\ell(w)-\ell\left(v_{i}\right)} E_{v_{i}}(t)=q+\cdots+q^{d_{i}-1}$ and $\mathbf{V}_{v_{i}}$ is a smooth point of $X_{w}$.

c) One has $H_{y, \pi}(t)=1+\sum_{i=1}^{|a|}\left(q+\cdots+q^{d_{i}}\right)=1+\sum_{s=1}^{d} a_{s}\left(q+\cdots+q^{s}\right)$.

d) $\mathbf{V}_{y}$ is a smooth point in each $X_{v_{i}}$.

Proof. First, one checks that $v_{i}(i)=1$ and $v_{i}(j)=w(j)$, for all $j \in[1,|a|+1] \backslash\{i\}$. From this one deduces that $X_{y} \subseteq X_{v_{i}} \subseteq X_{w}^{1}$, and that $v_{i} \not \leq v_{j}$ if $i \neq j$ (by comparing the $i$-th and $j$-th keys). Further, let $X_{z}$ be an irreducible component of $X_{w}^{1}$ and let $i=z^{-1}(1)$. Since $\mathbb{C}^{1} \subseteq \mathbf{V}_{z}^{|a|}$, one has $i \leq|a|$. From the definition of $v_{i}$, again, this implies that $z \leq v_{i}$. It follows that the $X_{v_{i}}$ are exactly the irreducible components of $X_{w}^{1}$. (In fact, the orbit $B \mathbf{V}_{v_{i}}$ is defined inside $X_{w}$ by the condition that $\mathbb{C}^{1} \subseteq V^{i}$ but $\mathbb{C}^{1} \nsubseteq V^{i-1}$.) Further, using 1.2(†) plus the fact that, for $\left.j \in\right] i, n-d_{i}[$ one has $w(j) \geq w\left(n-d_{i}-1\right)>w(i)$, one obtains that

$$
w \rightarrow w s_{n-2} \rightarrow \cdots \rightarrow w s_{n-2} \cdots s_{n-d_{i}} \rightarrow w s_{n-2} \cdots s_{n-d_{i}}\left(i, n-d_{i}\right)=v_{i} .
$$

Therefore, $\ell\left(v_{i}\right)=\ell(w)-d_{i}$. This proves assertion a).

b) Let us now describe the fibre $\pi^{-1}\left(\mathbf{V}_{v_{i}}\right)$. First, note that $\mathbb{C}^{i+d_{i}+1} \cap \mathbf{V}_{v_{i}}^{n-1}$ has dimension $d_{i}+i$. Second, it is easily seen that the natural projection

$$
p_{i}: \pi^{-1}\left(\mathbf{V}_{v_{i}}\right) \rightarrow \mathbb{P}\left(\left(\mathbb{C}^{i+d_{i}+1} \cap \mathbf{V}_{v_{i}}^{n-1}\right) / \mathbf{V}_{v_{i}}^{i}\right) \cong \mathbb{P}^{d_{i}-1}, \quad\left(U^{2}, \ldots, U^{a+1}\right) \mapsto U^{i+1},
$$

is surjective. But, clearly, for $j<i$, one has $U^{j+1}=\mathbb{C}^{1} \oplus \mathbf{V}_{v_{i}}^{j}$ (since $\mathbb{C}^{1} \nsubseteq \mathbf{V}_{v_{i}}^{i-1}$ ). Further, for every $j \in[i+1,|a|]$, one has $\mathbf{V}_{v_{i}}^{j} \nsubseteq \mathbb{C}^{d_{j-1}+j}$, whence $\mathbf{V}_{v_{i}}^{j} \neq U^{j}$, and therefore $U^{j+1}=U^{j}+\mathbf{V}_{v_{i}}^{j}$. This shows that $p_{i}$ is bijective, whence an isomorphism by Zariski's main theorem. This proves the first part of assertion b). On the other hand, since $H_{z, \pi}=1=P_{z, w}$ for all $\left.\left.z \in\right] v_{i}, w\right]$, by Lemma 2.2 and assertion a), then (1) gives

$$
H_{v_{i}, \pi}(t)=P_{v_{i}, w}(q)+t^{d_{i}} E_{v_{i}}(t) .
$$

The second part of b) is then an immediate consequence of this formula. Indeed, this is clear if $d_{i}=1$, so we may suppose that $d_{i} \geq 2$. Then, since $H_{v_{i}, \pi}-P_{v_{i}, w}$ has nonnegative coefficients and $\operatorname{deg} P_{v_{i}, w} \leq\left(d_{i}-1\right) / 2<d_{i}-1$, it follows that $P_{v_{i}, w}=1+\cdots+q^{s-1}$, for some $s \in\left[1, d_{i}-1\right]$. Therefore,

$$
E_{v_{i}}(t)=t^{-d_{i}}\left(q^{s}+\cdots+q^{d_{i}-1}\right)=t^{2 s-d_{i}}+\cdots+t^{d_{i}-2},
$$

and since $E_{v_{i}}\left(t^{-1}\right)=E_{v_{i}}(t)$, this implies that $s=1$, whence $t^{d_{i}} E_{v_{i}}(t)=q+\cdots+$ $q^{d_{i}-1}$ and $P_{v_{i}, w}=1$. Thus, by [5], $\mathbf{V}_{v_{i}}$ is a smooth point of $X_{w}$. 
Let us prove assertion c). The fibre $\pi^{-1}\left(\mathbf{V}_{y}\right)$ is the variety of flags $U^{2} \subset \cdots \subset$ $U^{|a|+1}$ which satisfy the following relations of inclusion:

$$
\left\{\begin{array}{llllllll}
\mathbb{C}^{1} & \subset \cdots \subset & \mathbf{V}_{y}^{i} & \subset \cdots \subset & \mathbf{V}_{y}^{|a|} & \subset \cdots \subset & \mathbf{V}_{y}^{n-2} \\
\cap & & & \cap & \cap & \cap & \\
U^{2} & \subset \cdots \subset & U^{i+1} & \subset \cdots \subset & U^{|a|+1} & & \cap \\
\cap & & & \cap & & & \\
\mathbb{C}^{d_{1}+2} & \subset \cdots \subset & \mathbb{C}^{i+d_{i}+1} & \subset \cdots \subset & \mathbb{C}^{n-1} & = & \mathbf{V}_{y}^{n-1}
\end{array}\right\} .
$$

Observe that, for $i=2, \ldots,|a|, \mathbf{V}_{y}^{i}$ is the $\mathbb{C}$-span of $e_{1}, e_{d_{1}+2}, \ldots, e_{d_{i-1}+i}$ and, therefore, one has $\mathbf{V}_{y}^{i} \subseteq \mathbb{C}^{d_{i-1}+i}$ but $\mathbf{V}_{y}^{i} \nsubseteq \mathbb{C}^{d_{i-1}+i-1}$.

We claim that $\pi^{-1}\left(\mathbf{V}_{y}\right)$ is the disjoint union of locally closed subvarieties $Y_{1}, \ldots$, $Y_{|a|}$ (with $Y_{1}$ open and $Y_{|a|}$ closed), such that, for $i<|a|, Y_{i}$ is isomorphic to $\mathbb{P}^{d_{i}} \backslash\{p t\}$, whereas $Y_{|a|} \cong \mathbb{P}^{d}$. Of course, this claim immediately implies assertion c).

Let $Y_{1}$ be the open subvariety of $\pi^{-1}\left(\mathbf{V}_{y}\right)$ consisting of those flags $\left(U^{2}, \ldots, U^{|a|+1}\right)$ such that $U^{2} \neq \mathbf{V}_{y}^{2}$. Let us prove that $Y_{1} \cong \mathbb{P}^{d_{1}} \backslash\{p t\}$. Let $\varphi$ denote the restriction to $Y_{1}$ of the natural projection from $\pi^{-1}\left(\mathbf{V}_{y}\right)$ to $\mathbb{P}\left(\mathbb{C}^{d_{1}+2} / \mathbb{C}^{1}\right) \cong \mathbb{P}^{d_{1}}$, which sends $\left(U^{2}, \ldots, U^{|a|+1}\right)$ to $U^{2}$. Clearly, $\varphi$ maps $Y_{1}$ onto $\mathbb{P}^{d_{1}} \backslash\{p t\}$. Thus, by Zariski's main theorem, it suffices to prove that $\varphi$ is injective. The hypothesis that $U^{2} \neq \mathbf{V}_{y}^{2}$ implies that $U^{3}=\mathbf{V}_{y}^{2}+U^{2}$, whence $U^{3} \subseteq \mathbb{C}^{d_{1}+2}$. Thus, since $\mathbf{V}_{y}^{3} \nsubseteq \mathbb{C}^{d_{2}+2}$, it follows that $\mathbf{V}_{y}^{3} \neq U^{3}$. Since $\mathbf{V}_{y}^{i} \subseteq \mathbb{C}^{d_{i-1}+i}$ but $\mathbf{V}_{y}^{i} \nsubseteq \mathbb{C}^{d_{i-1}+i-1}$, for all $i \in[2,|a|]$, one can repeat this argument to conclude that $U^{i} \neq \mathbf{V}_{y}^{i}$ and $U^{i+1}=\mathbf{V}_{y}^{i}+U^{2}$, for all $i \in[2,|a|]$. This proves that $\varphi$ is bijective, and hence that $Y_{1} \cong \mathbb{P}^{d_{1}} \backslash\{p t\}$.

Now, for $i=2, \ldots,|a|$, let $Y_{i}$ denote the locally closed subvariety consisting of those flags $\left(U^{2}, \ldots, U^{|a|+1}\right)$ such that $U^{j+1}=\mathbf{V}_{y}^{j+1}$ for $j<i$ and $U^{i+1} \neq \mathbf{V}_{y}^{i+1}$ (this last condition being omitted if $i=|a|$ ). Using an argument similar to the above, one deduces that $Y_{i} \cong \mathbb{P}^{d_{i}} \backslash\{p t\}$, for $i<|a|$, whereas $Y_{|a|} \cong \mathbb{P}^{d}$, and that $\pi^{-1}\left(\mathbf{V}_{y}\right)$ is the disjoint union of the $Y_{i}$. It follows that

$$
H_{y, \pi}=1+\sum_{i=1}^{|a|}\left(q+\cdots+q^{d_{i}}\right)=1+\sum_{s=1}^{d} a_{s}\left(q+\cdots+q^{s}\right) .
$$

This proves assertion c).

Finally, let us prove assertion d). From the definition of $v_{i}$, one derives easily that $X_{v_{i}}$ admits a resolution of singularities $\phi: Z_{v_{i}} \rightarrow X_{v_{i}}$, where $Z_{v_{i}}$ is the variety of pairs of flags $\left(\left(V^{k}\right)_{1 \leq k<n},\left(U^{j}\right)_{2 \leq j<i}\right)$ satisfying the following relations of inclusion:

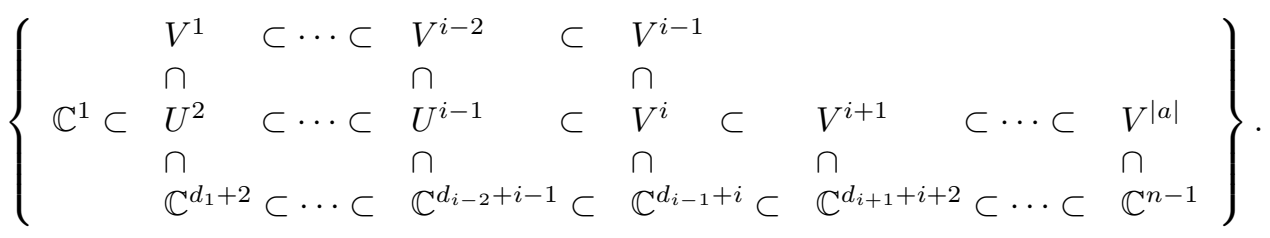

It is easily seen that $\phi$ is bijective over the open subvariety $\Omega$ of $X_{v_{i}}$ defined by the condition that $\operatorname{dim}\left(V^{i} \cap \mathbb{C}^{d_{j}+j+1}\right) \leq j+1$, for $j=1, \ldots, i-2$. In fact, this condition implies that $U^{j+1}=\mathbb{C}^{d_{j}+j+1} \cap V^{i}$ for all $j \in[1, i-2]$. Since $\phi$ is proper and $X_{v_{i}}$ normal, $\phi$ is an isomorphism over $\Omega$, by Zariski's main theorem. But, clearly, $\mathbf{V}_{y}$ 
belongs to $\Omega$. Therefore, $\mathbf{V}_{y}$ is a smooth point of $X_{v_{i}}$. This completes the proof of Proposition 2.4.

2.5. It follows from the previous proposition that

$$
H_{y, \pi}-\sum_{i=1}^{|a|} t^{\ell(w)-\ell\left(v_{i}\right)} E_{v_{i}}(t) P_{y, v_{i}}=P .
$$

Therefore, by (1), $P-P_{y, w}$ has nonnegative coefficients and the sought-for equality $P_{y, w}=P$ is equivalent to the vanishing of $E_{v}$ for all $v \in\left[y, w\left[\backslash\left\{v_{1}, \ldots, v_{|a|}\right\}\right.\right.$.

On the other hand, by [9, Cor. 4], $P_{y, w}-P_{z, w}$ has nonnegative coefficients, for all $z \in[y, w]$. Therefore, in order to prove the equality $P_{y, w}=P$, it suffices to exhibit, for all $s \in[1, d]$, an element $z_{s}$ in $[y, w]$ such that $P_{z_{s}, w}=1+a_{s} q^{s}$. We construct $z_{s}$ as follows.

Let $f_{s}=\sum_{i<s} a_{i}$ and $z_{s}=w(n-1, n-s-1)(n-1, n) \sigma_{s}$, where $\sigma_{s}$ is the $\left(a_{s}+1\right)$ cycle which transforms $\left(f_{s}+1, \ldots, f_{s}+a_{s}, n-s-1\right)$ into $\left(n-s-1, f_{s}+1, \ldots, f_{s}+a_{s}\right)$. We shall prove that

$$
P_{z_{s}, w}=1+a_{s} q^{s} .
$$

As observed above, this will complete the proof of the theorem.

2.6. Let $s \in[1, d]$. Set $h_{s}=w(n-s-1)=f_{s+1}+s+2$. One observes that $z_{s}(i)=w(i)$ if $i \leq f_{s}$, and $z_{s}^{-1}(j)=w^{-1}(j)$ if $j>h_{s}$. Let $F_{s}=\left[f_{s}+1, n\right] \cap$ $w^{-1}\left[1, h_{s}\right]=\left[f_{s}+1, n\right] \cap z_{s}^{-1}\left[1, h_{s}\right]$. From the definition of $w$, it follows that $F_{s}=\left[f_{s}+1, f_{s}+a_{s}\right] \sqcup[n-s-1, n]$. In particular, $\# F_{s}=a_{s}+s+2:=m_{s}$. Let $\phi\left(\right.$ resp. $\psi$ ) be the unique increasing bijection from $\left[1, m_{s}\right]$ to $F_{s}$ (resp. to $w\left(F_{s}\right)=z_{s}\left(F_{s}\right)$ ). Set $\bar{w}=\left.\psi^{-1} \circ w\right|_{F_{s}} \circ \phi$, and define $\overline{z_{s}}$ similarly. (Note that $\bar{w}$ depends on $s$; in order not to burden the notation we omit to indicate this.) Then one has the following proposition (see Section 4 for a geometric interpretation of this result in a more general setting).

Proposition. One has $P_{z_{s}, w}=P_{\overline{z_{s}}, \bar{w}}$.

Proof. Let us first recall the following known lemma, which is obtained easily by comparing the element $C_{u}^{\prime}$ defined in [11, (1.1.c)] with $C_{v}^{\prime} C_{x^{\prime}}^{\prime}$.

Lemma. Let $W$ be a Coxeter group and $W^{\prime}$ a parabolic subgroup. Let $x \leq u$ in $W$ and suppose that $x W^{\prime}=u W^{\prime}$ (resp. $\left.W^{\prime} x=W^{\prime} u\right)$. Let $v$ be the unique minimal element in this coset and set $u^{\prime}=v^{-1} u, x^{\prime}=v^{-1} x$ (resp. $u^{\prime}=u v^{-1}$ and $\left.x^{\prime}=x v^{-1}\right)$. Then $P_{x, u}=P_{x^{\prime}, u^{\prime}}$.

Now, let us show that the proposition is a consequence of the lemma. Let us write, for simplicity, $f, z, h, F, m$ instead of $f_{s}, z_{s}, h_{s}, F_{s}, m_{s}$. Let us denote by $W_{I}$ the subgroup of permutations of $[f+1, n]$. Then $w W_{I}=z W_{I}$. Let $v$ be the unique minimal element in this coset, and $w_{1}=v^{-1} w, z_{1}=v^{-1} z$. By the lemma, one has $P_{z, w}=P_{z_{1}, w_{1}}$.

Observe that the restriction of $v$ to the interval $[f+1, n]$ is precisely the unique increasing bijection from $[f+1, n]$ to $w([f+1, n])$. Thus, recalling that $F$ denotes $[f+1, n] \cap w^{-1}[1, h]$, one deduces that $v^{-1}$ induces an increasing bijection from $w(F)$ to an interval $[f+1, g]$, for some $g \leq n$. Denoting by $\eta$ the unique increasing bijection from $[1, m]$ to $[f+1, g]$, it follows that $\psi^{-1}=\eta^{-1} \circ v^{-1}$. Therefore, $\bar{w}=\left.\eta^{-1} \circ v^{-1} \circ w\right|_{F} \circ \phi$. 
Further, denoting by $W_{K}$ the subgroup of permutations of $[f+1, g]$, the hypotheses also imply that $W_{K} w_{1}=W_{K} z_{1}$. Let $\theta$ be the unique minimal element in this coset, and let $w_{2}=w_{1} \theta^{-1}$ and $z_{2}=z_{1} \theta^{-1}$. On the one hand, by the lemma again, one has $P_{z_{1}, w_{1}}=P_{z_{2}, w_{2}}$ and, therefore, $P_{z, w}=P_{z_{2}, w_{2}}$. On the other hand, one observes that $\theta^{-1}$ is the unique increasing bijection from $[f+1, g]$ to $F$. One deduces that $\phi=\theta^{-1} \circ \eta$, and hence that $\bar{w}=\left.\eta^{-1} \circ v^{-1} \circ w\right|_{F} \circ \theta^{-1} \circ \eta$.

But $\left.v^{-1} \circ w\right|_{F} \circ \theta^{-1}$ identifies with $w_{2}$, and therefore one obtains that $\bar{w}=$ $\eta^{-1} \circ w_{2} \circ \eta$, and similarly for $\bar{z}$ and $z_{2}$. Since the map $x \mapsto \eta^{-1} \circ x \circ \eta$ induces an order-preserving isomorphism of Coxeter groups $S_{[1, m]} \cong S_{[f+1, g]}$, it follows that $P_{z_{2}, w_{2}}=P_{\bar{z}, \bar{w}}$. The proposition is proved.

2.7. Again, let $s \in[1, d]$ be fixed. It follows from the definitions that the codes of $\bar{w}$ and $\overline{z_{s}}$ are

$$
c(\bar{w})=\left((s+1)^{a_{s}+1}, s, s-1, \ldots, 2,0,0\right) \quad \text { and } \quad c\left(\overline{z_{s}}\right)=\left(0, s^{a_{s}}, s-1, \ldots, 1,0,0\right) .
$$

Thus, $\overline{z_{s}}<\bar{w}$ is the pair associated to the polynomial $1+a_{s} q^{s}$. Therefore, in order to complete the proof of the theorem in general, it suffices to do so in the special case where $P-1$ is a monomial, since, by Proposition 2.6, this will imply the sought-for equality 2.5.(2).

\section{Completion of the proof in the monomial CASE}

In this section, let $a, d$ be positive integers, $n=a+d+2$, and $P=1+a q^{d}$. Let $w>y$ be the elements of $S_{n}$ associated to $P$ in 2.1 (see also 1.4). In fact, one has $w(i)=i+1+d$ for $i \leq a+1, w(i)=n+1-i$ for $i \in[a+2, n-2]$ (this condition being void if $d=1), w(n-1)=1$ and $w(n)=2$, whereas $y(1)=1, y(i)=w(i-1)$ for $i \in[2, a+1], y(i)=w(i)$ for $i \in[a+2, n-2], y(n-1)=2$ and $y(n)=n$. Further, $\ell(w)-\ell(y)=2 d+a$.

3.1. The notation and results of the previous section apply in this special case. In particular, with notation as in Proposition 2.4, we know already that $H_{y, \pi}(t)=$ $1+a\left(q+\cdots+q^{d}\right)$ and that $t^{d} E_{v_{i}}(t)=q+\cdots+q^{d-1}$, for $i=1, \ldots, a$. Therefore, one has

$$
1+a q^{d}=P_{y, w}+\sum_{\substack{v \in\left[y, w\left[ \\v \notin\left\{v_{1}, \ldots, v_{a}\right\}\right.\right.}} t^{\ell(w)-\ell(v)} E_{v}(t) P_{y, v} .
$$

Further, using the fact that the terms in the right-hand side have nonnegative coefficients and that $P_{y, w}$ and $P_{y, v}$ have constant term equal to 1 , one deduces that if $E_{v}(t) \neq 0$ for some $v$ in the above sum, then, necessarily, $P_{y, v}=1$ and $t^{\ell(w)-\ell(v)} E_{v}(t)=c q^{d}$ for some $c \in[1, a]$. Since $E_{v}(t)=E_{v}\left(t^{-1}\right)$, this implies that $\ell(w)-\ell(v)=2 d$. Therefore, the sought-for equality $P_{y, w}=1+a q^{d}$ is a consequence of the following proposition.

Proposition. For all $v \in[y, w]$ such that $\ell(w)-\ell(v)=2 d$, one has $\operatorname{deg}_{t} H_{v, \pi}<2 d$, and hence $E_{v}=0$. In fact, one has more precisely $H_{v, \pi}=1+b\left(q+\cdots+q^{d-1}\right)$, where $b=\#\left\{i \in[1, a] \mid v \leq v_{i}\right\}$.

Proof. Recall first that, if $X_{v} \nsubseteq X_{w}^{1}$, that is, if $v^{-1}(1)>a$, then $H_{v, \pi}=1$, by Lemma 2.2. Thus, we may assume that $v^{-1}(1) \leq a$. We will need the following lemma. 
Lemma. Let $v \in[y, w]$ be such that $v^{-1}(1)=i \leq a$. If $\mathbb{C}^{d+i+1} \subseteq \mathbf{V}_{v}^{n-1}$, then $\ell(w)-\ell(v) \geq 2 d+1$.

Proof. Since $v \leq w$, then $v(j) \leq j+d+1$, for $j=1, \ldots, a$. Further, we assume that $v^{-1}(1)=i \leq a$. On the other hand, the hypothesis $\mathbb{C}^{d+i+1} \subseteq \mathbf{V}_{v}^{n-1}$ is equivalent to $v(n)>d+i+1$. Thus, one has $v(n)=d+j+1$ for some $j \in[i+1, a+1]$.

One then deduces that $v$ is smaller than the permutation $w_{i, j}$ defined by $w_{i, j}(k)=$ $w(k)$ if $k \notin\{i, j, n-1, n\}$, while $w_{i, j}(i)=1, w_{i, j}(j)=d+i+1, w_{i, j}(n-1)=2$ and $w_{i, j}(n)=d+j+1$. Let $u_{i, j}=v_{i}(j, a+2)$. Using $1.2(\dagger)$, it is easily seen that

$$
v_{i} \rightarrow u_{i, j} \rightarrow u_{i, j} s_{a+2} \rightarrow \cdots \rightarrow u_{i, j} s_{a+2} \cdots s_{n-1}=w_{i, j}
$$

Thus, $\ell\left(v_{i}\right)-\ell\left(w_{i, j}\right)=d+1$ and hence $\ell(w)-\ell\left(w_{i, j}\right)=2 d+1$. The lemma is proved.

Let us now prove the proposition. Let $v \in W$ be such that $y \leq v \leq w$ and $v^{-1}(1):=i_{1} \leq a$. These hypotheses, together with the fact that $w([1, j])=$ $[d+2, d+j+1]$ and $y([1, j])=\{1\} \cup[d+2, d+j]$ for $j=1, \ldots, a$, imply that there exists a sequence $i_{1}<\cdots<i_{b}$ such that $v(j)=j+d+1$ for $j \in[1, a] \backslash\left\{i_{1}, \ldots, i_{b}\right\}$ while $v\left(i_{r}\right)=i_{r-1}+d+1$ for $r=2, \ldots, b$ (this condition being empty if $b=1$ ). In particular, one has $v([1, a])=\{1\} \cup[d+2, n-1] \backslash\left\{i_{b}+d+1\right\}$. Set $J=\left\{i_{1}, \ldots, i_{b}\right\}$. Then, clearly, $J=\left\{j \in[1, a] \mid \mathbf{V}_{v}^{j} \subseteq \mathbb{C}^{d+j}\right\}$ and one has, for every $j \in[1, a]$ :

$$
\operatorname{dim}\left(\mathbf{V}_{v}^{a} \cap \mathbb{C}^{d+j+1}\right)= \begin{cases}j+1 & \text { if } j<i_{b} \\ j & \text { if } j \geq i_{b}\end{cases}
$$

Further, it follows from the definition of the $v_{i}$ 's (see 2.4) that $v \leq v_{j}$ if $j \in J$; conversely, since $\mathbf{V}_{v_{i}}^{i} \subseteq \mathbb{C}^{d+i}$, then $v \not \leq v_{i}$ if $i \notin J$. Therefore, one also has $J=$ $\left\{j \in[1, a] \mid v \leq v_{j}\right\}$.

Suppose, moreover, that $\ell(w)-\ell(v) \leq 2 d$. Then, by the previous lemma, we have $\mathbb{C}^{d+i_{1}+1} \nsubseteq \mathbf{V}_{v}^{n-1}$ and hence

$$
\operatorname{dim}\left(\mathbb{C}^{d+j+1} \cap \mathbf{V}_{v}^{n-1}\right)=d+j, \quad \forall j \in\left[i_{1}, a\right] .
$$

Now, we claim that the fibre $\pi^{-1}\left(\mathbf{V}_{v}\right)$ is the disjoint union of locally closed subvarieties $Y_{i_{1}}, \ldots, Y_{i_{b}}$ (with $Y_{i_{1}}$ open and $Y_{i_{b}}$ closed), such that $Y_{i_{r}}$ is isomorphic to $\mathbb{P}^{d-1} \backslash\{p t\}$ for $r<b$, whereas $Y_{i_{b}} \cong \mathbb{P}^{d-1}$.

In fact, set $U^{1}=\mathbb{C}^{1}$ and for every $j=2, \ldots, a+1$, let $Y_{j}$ denote the locally closed subvariety of $\pi^{-1}\left(\mathbf{V}_{v}\right)$ consisting of those flags $\left(U^{\bullet}\right)$ such that $U^{j-1} \subseteq \mathbf{V}_{v}^{a}$ and $U^{j} \nsubseteq \mathbf{V}_{v}^{a}$. Then the $Y_{j}^{\prime} s$ are clearly disjoint and, since $U^{1}=\mathbb{C}^{1} \subseteq \mathbf{V}_{v}^{a}$ but $U^{a+1} \nsubseteq \mathbf{V}_{v}^{a}$, they form a partition of $\pi^{-1}\left(\mathbf{V}_{v}\right)$.

Next, observe that if $j \notin J$, then $\mathbf{V}_{v}^{j} \neq U^{j}$ (since $\mathbf{V}_{v}^{j} \nsubseteq \mathbb{C}^{j+d}$ ) and hence $U^{j+1}=U^{j}+\mathbf{V}_{v}^{j}$. Therefore, $Y_{j}=\emptyset$ if $j \notin J$. Now, let us prove that the $Y_{i_{r}}$, for $r=1, \ldots, b$, are as we claimed above.

Let $r \in[1, b]$. Suppose that $\left(U^{\bullet}\right) \in Y_{i_{r}}$. Then, clearly, one has $U^{j+1}=U^{i_{r}+1}+$ $\mathbf{V}_{v}^{j}$ for $j \in\left[i_{r}, a\right]$. Secondly, one has $U^{i_{r}}=\mathbf{V}_{v}^{i_{r}}$ (for otherwise one would have $\left.U^{i_{r}+1}=U^{i_{r}}+\mathbf{V}_{v}^{i_{r}} \subseteq \mathbf{V}_{v}^{a}\right)$ and hence, using $(*)$, one obtains that $U^{j}=U^{i_{r}} \cap \mathbb{C}^{j+d}$ for $j \leq i_{r}$. Conversely, this shows that the projection $p_{i_{r}}:\left(U^{\bullet}\right) \mapsto U^{i_{r}}$, from $Y_{i_{r}}$ to the projective space of $\left(\mathbb{C}^{i_{r}+1+d} \cap \mathbf{V}_{v}^{n-1}\right) / \mathbf{V}_{v}^{i_{r}}$ is a bijection onto its image.

Further, since $\operatorname{dim}\left(\mathbb{C}^{i_{r}+1+d} \cap \mathbf{V}_{v}^{n-1}\right) / \mathbf{V}_{v}^{i_{r}}=d$, by $(* *)$, whereas

$$
\operatorname{dim}\left(\mathbb{C}^{i_{r}+1+d} \cap \mathbf{V}_{v}^{a}\right) / \mathbf{V}_{v}^{i_{r}}= \begin{cases}1 & \text { for } r<b \\ 0 & \text { for } r=b\end{cases}
$$


by $(*)$, it follows that the image of $p_{i_{r}}$ is $\mathbb{P}^{d-1} \backslash\{p t\}$ when $r<b$, and $\mathbb{P}^{d-1}$ when $r=b$. The claim then follows, by Zariski's main theorem, and one deduces that $H_{z, \pi}(q)=1+b\left(q+\cdots+q^{d-1}\right)$.

This completes the proof of Proposition 3.1 and, therefore, of Theorem 2.1.

Remark. Along the same lines as above, one can prove, using [5], that the $w_{i, j}$ defined in the proof of Lemma 3.1 (for $1 \leq i<j \leq a+1$ ) are exactly the irreducible components of the singular locus of $X_{w}$, and that the corresponding KazhdanLusztig polynomials are all equal to $1+q^{d}$. We leave this to the interested reader.

3.2. Let us now return to the general case; that is, let $y<w$ be as in 2.1. Since Theorem 2.1 is proved, then, by the discussion at the beginning of 2.5 , we have also shown that $E_{v}=0$ for all $v \in\left[y, w\left[\backslash\left\{v_{1}, \ldots, v_{|a|}\right\}\right.\right.$. Therefore, denoting by $j$ the inclusion of the open subset $C_{[y, w]}$ in $X_{w}$, one obtains that

$$
j^{*} R \pi_{*}(\mathbb{C}[\ell(w)]) \cong j^{*} I C\left(X_{w}\right) \oplus \bigoplus_{i=1}^{|a|} H^{\bullet}\left(\mathbb{P}^{d_{i}-2}\right)\left[d_{i}-2\right] \otimes j^{*} I C\left(X_{v_{i}}\right) .
$$

Note that $H^{\bullet}\left(\mathbb{P}^{d-2}\right)[d-2]$ equals 0 if and only if $d=1$, and equals 0 in degrees $\neq 0$ if and only if $d \leq 2$. Thus $\pi$ is small (resp. semi-small) over $C_{[y, w]}$ if and only if $d=1$ (resp. $d \leq 2$ ).

3.3. Let us conclude this section with the following remark. First, if $W_{1}, W_{2}$ are Coxeter groups, $W=W_{1} \times W_{2}$, and $y_{i} \leq w_{i}$ in $W_{i}$, for $i=1,2$, then it is known (and easy to check) that $P_{y_{1} y_{2}, w_{1} w_{2}}=P_{y_{1}, w_{1}} P_{y_{2}, w_{2}}$.

Now, let $P_{1}, P_{2} \in 1+q \mathbb{N}[q]$ and let $P=P_{1} P_{2}$. Set $a_{i}=P_{i}(1)-1, a=a_{1}+a_{2}$ and $d=\operatorname{deg} P$. Then the pair $y_{P}<w_{P}$ belongs to $S_{n(P)}$, where $n(P)=1+d+P(1)=$ $2+d+a+a_{1} a_{2}$, and satisfies $\ell\left(w_{P}\right)-\ell\left(y_{P}\right)=2 d+a+a_{1} a_{2}$. On the other hand, let $n^{\prime}(P)=n\left(P_{1}\right)+n\left(P_{2}\right)=4+d+a$. Then, the elements $y_{P}^{\prime}:=y_{P_{1}} y_{P_{2}}$ and $w_{P}^{\prime}:=w_{P_{1}} w_{P_{2}}$ belong to $S_{n\left(P_{1}\right)} \times S_{n\left(P_{2}\right)} \subset S_{n^{\prime}(P)}$ and, by the above, one has $P_{y_{P}^{\prime}, w_{P}^{\prime}}=P$. This is an improvement on the general construction, in the sense that $\ell\left(w_{P}^{\prime}\right)-\ell\left(y_{P}^{\prime}\right)=2 d+a<\ell\left(w_{P}\right)-\ell\left(y_{P}\right)$.

\section{A focalization theorem}

In this section, which is independent of the previous ones, we study certain open neighbourhoods in Schubert varieties and prove an isomorphism which provides, in a more general setting, a geometric explanation for the equality in Proposition 2.6.

4.1. Let $k$ be an algebraically closed field of arbitrary characteristic, $G$ a connected semi-simple algebraic group over $k, T$ a maximal torus inside a Borel subgroup $B$, $W$ the Weyl group, $R$ the root system, and $\Delta$ (resp. $R^{+}$) the set of simple (resp. positive) roots associated with $B$. Let $U$ (resp. $U^{-}$) be the unipotent radical of $B$ (resp. of the opposite Borel subgroup). For $w \in W$, we denote by $e_{w B}$ the point $w B / B$ in $G / B$ and by $C_{w}$ its $B$-orbit (Bruhat cell). Then the Schubert variety $X_{w}$ is the closure of $C_{w}$. Let $\leq$ denote the Bruhat-Chevalley order on $W$.

Suppose that $y \leq w$. Set $\mathcal{V}_{y, w}=y\left(U^{-}\right) e_{y B} \cap X_{w}$. This is a $T$-stable, open affine neighbourhood of $y$ in $X_{w}$. In fact, since $e_{y B}$ is the unique closed $T$-orbit in $y\left(U^{-}\right) e_{y B}$, one can show that $\mathcal{V}_{y, w}$ is the unique $T$-stable, open affine neighbourhood of $y$ in $X_{w}$, see [4, Lemma 2.4]. Set $\mathcal{N}_{y, w}=\left(y\left(U^{-}\right) \cap U^{-}\right) e_{y B} \cap X_{w}$. This is a closed, $T$-stable, subvariety of $\mathcal{V}_{y, w}$. Recall from [11, Lemma A4.(b)] the isomorphism $\mathcal{V}_{y, w} \cong C_{y} \times \mathcal{N}_{y, w}$. 
We shall denote by $C_{[y, w]}$ (resp. $B[y, w] B$ ) the union of the Bruhat cells $C_{z}$ (resp. of the double cosets $B z B$ ), for $z \in[y, w]$. This is an open subvariety of $X_{w}$ (resp. of $\overline{B w B}$, the closure of $B w B$ in $G$ ). Further, it is well known, and easy to verify, that $\mathcal{V}_{y, w} \subseteq C_{[y, w]}$.

4.2. If $I$ is a subset of $\Delta$, let $R_{I}$ be the corresponding root system, and $W_{I}$ and $P_{I}$ the corresponding parabolic subgroups of $W$ and $G$.

Let $w \in W$ and let $v$ be the unique minimal element in the $\operatorname{coset} w W_{I}$. It is well known that the map $z \mapsto v z$ induces an isomorphism of posets $W_{I} \cong w W_{I}$, and that $\ell(v z)=\ell(v)+\ell(z)$, for all $z \in W_{I}$. We shall need to describe similarly the intersection $w W_{I} \cap W_{J} w$, where $J$ is a second, arbitrary, subset of $\Delta$.

Proposition. Let $I, J$ be subsets of $\Delta$ and let $w \in W$. Let $v$ be the minimal element in the coset $w W_{I}$, let $K=I \cap v^{-1} R_{J}$ and $w_{1}=v^{-1} w$, and let $\theta$ be the minimal element in the coset $W_{K} w_{1}$. Then the map $\psi: W_{K} \rightarrow w W_{I} \cap W_{J} w$, $z \mapsto v z \theta$ is an isomorphism of posets. Further, one has $\ell(v z \theta)=\ell(v)+\ell(z)+\ell(\theta)$, for all $z \in W_{K}$.

This is certainly known; yet since we are not aware of a reference, we give a proof, which begins with the following lemma. Set $v^{-1} W_{J} v=v^{-1}\left(W_{J}\right)$.

Lemma. One has $W_{I} \cap v^{-1}\left(W_{J}\right)=W_{K}$.

Proof. It suffices to prove that $v\left(W_{I}\right) \cap W_{J}$ is generated by the reflections $s_{\beta}$, for $\beta \in v(I) \cap R_{J}$. Denote by $H$ the subgroup generated by these reflections. Let $w \in v\left(W_{I}\right) \cap W_{J}$ and let $w=v s_{\alpha_{1}} \cdots s_{\alpha_{r}} v^{-1}$ be a reduced decomposition in $v\left(W_{I}\right)$ (that is, $\alpha_{i} \in I$ ). We prove by induction on $r$ that $v \alpha_{i} \in R_{J}$ for all $i$, and hence that $w \in H$. This is immediate if $r=1$, so assume that $r \geq 2$. Set $N(w)=\left\{\gamma \in R^{+} \mid w \gamma \in R^{-}\right\}$. Since $w \in W_{J}$, then $N(w) \subseteq R_{J}$. On the other hand, by the assumption on $v$, one has $v\left(R_{I}^{ \pm}\right) \subseteq R^{ \pm}$, and it follows that $v \alpha_{r} \in R^{+}$ and $w v \alpha_{r} \in R^{-}$. One deduces that $v \alpha_{r} \in R_{J}$. Therefore, $w s_{v \alpha_{r}} \in H$ and we are done by induction.

Let us then derive the proposition. First, one sees immediately that $\psi$ is an order isomorphism onto its image, and that the second assertion holds. Further, let $x \in w W_{I} \cap W_{J} w$ and $x_{1}=v^{-1} x$. Then $v x_{1} \in W_{J} v w_{1}$ and hence $x_{1} w_{1}^{-1} \in$ $v^{-1}\left(W_{J}\right) \cap W_{I}=W_{K}$. It follows that $\psi$ is surjective, and the proposition is proved.

Remark. The statement of the proposition is not symmetric in $I$ and $J$. But, of course, their roles can be reversed, as follows. Let $u$ be the minimal element in the coset $W_{J} w, K^{\prime}=J \cap u\left(R_{I}\right), w_{1}^{\prime}=w u^{-1}$, and $\gamma$ the minimal element in the coset $w_{1}^{\prime} W_{K^{\prime}}$. Then one obtains similarly that the map $\psi^{\prime}: W_{K^{\prime}} \rightarrow w W_{I} \cap W_{J} w, z^{\prime} \mapsto$ $\gamma z^{\prime} u$ is an isomorphism of posets.

Therefore, the map $\psi^{-1} \psi^{\prime}: z^{\prime} \mapsto v^{-1} \gamma z^{\prime} u \theta^{-1}$, from $W_{K^{\prime}}$ to $W_{K}$, is an isomorphism of posets. Further, since the neutral element of $W$ is the minimal element in both $W_{K}$ and $W_{K^{\prime}}$, one deduces that $u \theta^{-1}=\left(v^{-1} \gamma\right)^{-1}$ and, therefore, that $\psi^{-1} \psi^{\prime}$ is an isomorphism of Coxeter groups.

4.3. Let $y<w$ in $W$ and suppose that $y \in w W_{I} \cap W_{J} w$. Let $v$ be the unique minimal element in the coset $w W_{I}$ and let $w_{1}=v^{-1} w$ and $y_{1}=v^{-1} y$. Let $K=$ $I \cap v^{-1} R_{J}$ and let $\theta$ be the unique minimal element in the coset $W_{K} w_{1}$. Then, let $w_{2}=w_{1} \theta^{-1}$ and $y_{2}=y_{1} \theta^{-1}$. 
Theorem. There is a T-equivariant isomorphism

$$
C_{[y, w]} \cong C_{v} \times v\left(B\left[y_{2}, w_{2}\right] B \times{ }^{B} C_{\theta}\right) .
$$

Further, it induces a T-equivariant isomorphism $\mathcal{N}_{y, w} \cong v \mathcal{N}_{y_{2}, w_{2}}$.

Proof. First, let us introduce some notation. For $x \in W$ and $H$ a subgroup of $G$ normalized by $T$, let $x(H):=\dot{x} H \dot{x}^{-1}$, where $\dot{x}$ is any representative of $x$ in $N(T)$, the normalizer of $T$ in $G$. Then, let $U^{x}=U \cap x\left(U^{-}\right)$.

Set $Q=P_{I}$ and denote by $U_{Q}^{-}$the unipotent radical of the opposite parabolic subgroup. Let $\pi$ be the projection $G / B \rightarrow G / Q$ and let $e_{Q}=\pi\left(e_{B}\right)$. Then, by Bruhat's decomposition, the multiplication map $v\left(U_{Q}^{-}\right) \times v Q / B \rightarrow G / B,(u, x) \mapsto$ $u x$, induces an isomorphism $\phi_{0}: v\left(U_{Q}^{-}\right) \times v Q / B \cong \pi^{-1}\left(v U_{Q}^{-} e_{Q}\right)$. Note that $v\left(U_{Q}^{-}\right) \cap$ $U=U^{v}$ (since $v$ is minimal in its coset $v W_{I}$ ) and that, by Bruhat's decomposition again, one has $U^{v} \cong C_{v Q}$ via $u \mapsto u e_{v Q}$. Therefore, $\phi_{0}$ induces an isomorphism

$$
\phi_{1}: U^{v} \times v Q / B \rightarrow \pi^{-1}\left(C_{v Q}\right), \quad(u, x) \mapsto u x .
$$

Further, the hypothesis that $y \in w W_{I}$ implies that $C_{[y, w]}$ is contained in $\pi^{-1}\left(C_{v Q}\right)$. Since $C_{[y, w]}$ is $B$-stable, one deduces that $\phi_{1}$ restricts to a $T U^{v}$-equivariant isomorphism

$$
\phi: U^{v} \times\left(v Q / B \cap C_{[y, w]}\right) \stackrel{\cong}{\rightrightarrows} C_{[y, w]}, \quad(u, x) \mapsto u x .
$$

We claim that $v Q / B \cap C_{[y, w]}=v C_{\left[y_{1}, w_{1}\right]}$. In fact, let $z \in[y, w]$. Since $[y, w]=$ $v\left[y_{1}, w_{1}\right]$, then for any $z \in[y, w]$ one has $U^{z}=U^{v} v\left(U^{z_{1}}\right)$, where $z_{1}=v^{-1} z \in W_{I}$. This shows, first, that $v C_{\left[y_{1}, w_{1}\right]} \subseteq v Q / B \cap C_{[y, w]}$ and, second, that the restriction of $\phi$ to $U^{v} \times v C_{\left[y_{1}, w_{1}\right]}$ is surjective. Since $\phi$ is bijective, the claim follows. Therefore, we obtain a $T U^{v}$-equivariant isomorphism

$$
\phi: U^{v} \times v C_{\left[y_{1}, w_{1}\right]} \stackrel{\cong}{\rightarrow} C_{[y, w]}, \quad(u, x) \mapsto u x .
$$

Now, let us prove that $C_{\left[y_{1}, w_{1}\right]} \cong B\left[y_{2}, w_{2}\right] B \times{ }^{B} C_{\theta}$. Let $P_{K}$ be the parabolic subgroup corresponding to $K$, and let $w_{K}$ denote the longest element in $W_{K}$. By hypothesis, $y_{1}, w_{1} \in W_{K} \theta$ and hence, by Bruhat's decomposition, one has $B\left[y_{1}, w_{1}\right] B \subseteq P_{K} \theta B$. We claim that the morphism $\psi: P_{K} \times{ }^{B} C_{\theta} \rightarrow P_{K} C_{\theta}=$ $C_{\left[\theta, w_{K} \theta\right]}$ is an isomorphism.

Note first that the orbit $C_{\theta}$ (resp. $C_{w_{K} \theta}$ ) is closed (resp. open) in $C_{\left[\theta, w_{K} \theta\right]}$. Now, $\psi$ is proper, since it factors as the composite of the closed immersion $P_{K} \times{ }^{B} C_{\theta} \hookrightarrow$ $P_{K} \times{ }^{B} C_{\left[\theta, w_{K} \theta\right]}$ followed by the projection from $P_{K} \times{ }^{B} C_{\left[\theta, w_{K} \theta\right]} \cong\left(P_{K} / B\right) \times C_{\left[\theta, w_{K} \theta\right]}$ onto $C_{\left[\theta, w_{K} \theta\right]}$. Further, one deduces from Bruhat's decomposition, again, that $\psi$ maps bijectively $B z B \times \times^{B} C_{\theta}$ onto $C_{z \theta}$, for all $z \in W_{K}$, and that it induces an isomorphism $B w_{K} B \times{ }^{B} C_{\theta} \cong C_{w_{K} \theta}$ over the open orbit $C_{w_{K} \theta}$. Thus, $\psi$ is birational and bijective. Since $C_{\left[\theta, w_{K} \theta\right]}$ is normal $([15,14,1])$, it follows from Zariski's main theorem that $\psi$ is an isomorphism.

Moreover, since $\psi\left(B z B \times{ }^{B} C_{\theta}\right)=C_{z \theta}$ for all $z \in W_{K}$, and since multiplication by $\theta$ induces an isomorphism of Bruhat intervals $[x, z] \cong[x \theta, z \theta]$, one concludes that $\psi$ induces a $B$-equivariant isomorphism $B[x, z] B \times{ }^{B} C_{\theta} \cong C_{[x \theta, z \theta]}$, for all $x \leq z$ in $W_{K}$. Thus, one obtains, in particular, that

$$
C_{\left[y_{1}, w_{1}\right]} \cong B\left[y_{2}, w_{2}\right] B \times{ }^{B} C_{\theta} .
$$

This completes the proof of the first assertion of the theorem. 
Let us prove the second assertion. Let $p$ denote the fibration $\overline{B w_{2} B} \times{ }^{B} C_{\theta} \rightarrow X_{w_{2}}$ and let $\Omega^{\prime}=p^{-1}\left(\mathcal{V}_{y_{2}, w_{2}}\right)$, a $T$-stable open subvariety of $C_{\left[y_{1}, w_{1}\right]}$. Since, by Bruhat's decomposition, the fibration $\overline{B w_{2} B} \rightarrow X_{w_{2}}$ trivializes over $\mathcal{V}_{y_{2}, w_{2}}$, then so does $p$. Thus, there is a $T$-equivariant isomorphism $\Omega^{\prime} \cong \mathcal{V}_{y_{2}, w_{2}} \times C_{\theta}$. It follows that $\phi\left(U^{v} \times v \Omega^{\prime}\right)$ is an open, $T$-stable, affine neighbourhood of $e_{y B}$ in $C_{[y, w]}$. By [4, Lemma 2.4], this implies that $\phi\left(U^{v} \times v \Omega^{\prime}\right)=\mathcal{V}_{y, w}$.

Set $U(K)^{-}=U^{-} \cap P_{K}$ and $\mathcal{Z}_{y_{2}, w_{2}}=\left\{u \in y_{2}\left(U(K)^{-}\right) \cap U^{-} \mid u e_{y_{2} B} \in X_{w_{2}}\right\}$. Then, clearly, one has $T$-equivariant isomorphisms $\mathcal{Z}_{y_{2}, w_{2}} \cong \mathcal{N}_{y_{2}, w_{2}}, u \mapsto u e_{y_{2}}$ and

$$
U^{y_{2}} \times \mathcal{Z}_{y_{2}, w_{2}} \times y_{2}\left(U^{\theta}\right) \cong \Omega^{\prime}, \quad\left(u_{1}, u_{2}, u_{3}\right) \mapsto u_{1} u_{2} u_{3} e_{y_{1} B} .
$$

Thus, using (1), one obtains a $T$-equivariant isomorphism

$$
\sigma: U^{v} \times v\left(U^{y_{2}}\right) \times v\left(\mathcal{Z}_{y_{2}, w_{2}}\right) \times v y_{2}\left(U^{\theta}\right) \stackrel{\cong}{\rightrightarrows} \mathcal{V}_{y, w}, \quad\left(u_{1}, u_{2}, u_{3}, u_{4}\right) \mapsto u_{1} u_{2} u_{3} u_{4} e_{y B} .
$$

Therefore, it only remains to show that $\sigma\left(v\left(\mathcal{Z}_{y_{2}, w_{2}}\right)\right)=\mathcal{N}_{y, w}$. Since both are closed subvarieties of $\mathcal{V}_{y, w}$ of dimension $\ell(w)-\ell(y)$, it is enough to prove that $\sigma\left(v\left(\mathcal{Z}_{y_{2}, w_{2}}\right)\right) \subseteq \mathcal{N}_{y, w}$. Further, since $\sigma\left(v\left(\mathcal{Z}_{y_{2}, w_{2}}\right)\right) \subseteq X_{w}$, it suffices to prove that $v\left(y_{2}\left(U(K)^{-}\right) \cap U^{-}\right) \subseteq U^{-} \cap y\left(U^{-}\right)$.

Thus, let $\alpha \in y_{2}\left(R_{K}^{-}\right) \cap R^{-}$. Note that $s_{\alpha} y_{2}>y_{2}$ and $s_{\alpha} y_{2} \in W_{K}$. Since $v$ is minimal in its coset $v W_{I}$, then $v \alpha \in U^{-}$and, therefore, the condition $v \alpha \in y\left(R^{-}\right)$ is equivalent to $s_{v \alpha} y>y$. But one has $s_{v \alpha} y=v s_{\alpha} y_{2} \theta$, and hence, since $s_{\alpha} y_{2} \theta \in$ $W_{K} \theta \subseteq W_{I}$,

$$
\ell\left(s_{v \alpha} y\right)=\ell(v)+\ell\left(s_{\alpha} y_{2}\right)+\ell(\theta)>\ell(v)+\ell\left(y_{2}\right)+\ell(\theta)=\ell(y) .
$$

This completes the proof of the equality $\sigma\left(v\left(\mathcal{Z}_{y_{2}, w_{2}}\right)\right)=\mathcal{N}_{y, w}$ and, therefore, of the second assertion of the theorem.

4.4. Application to the symmetric group. Let $W=S_{n}$ and let $W_{I}=S_{[a, b]}$ and $W_{J}=S_{[c, d]}$, for some $a<b$ and $c<d$ in [1,n]. (We restrict ourselves to this case for simplicity, but, of course, the discussion below extends readily to the case of arbitrary Young subgroups.) Let $y<w$ in $S_{n}$ and suppose that $y(i)=w(i)$ if $i<a$ or $i>b$, and that $y^{-1}(j)=w^{-1}(j)$ if $j<c$ or $j>d$.

On the one hand, let $v, w_{1}, y_{1}, \theta, w_{2}, y_{2}$ be as in 4.3. On the other hand, note that $w[a, b]=y[a, b]$ and $w^{-1}[c, d]=y^{-1}[c, d]$ and let $F=[a, b] \cap w^{-1}[c, d]$ and $E=[c, d] \cap w[a, b]$. Then $w$ and $y$ induce bijections $\tilde{w}, \tilde{y}$ from $F$ to $E$, and these give rise in a natural manner to elements $\bar{w}, \bar{y}$ of $S_{m}$, where $m=\# F$. Explicitly, let $\phi($ resp. $\psi$ ) be the unique increasing bijection from $[1, m]$ to $F$ (resp. to $E$ ); then $\bar{w}=\psi^{-1} \circ \tilde{w} \circ \phi$, and similarly for $\bar{y}$ (compare 2.6).

Further, as in 2.6, one observes that $v^{-1}$ induces an increasing bijection from $E$ to some interval $[f, g]$, where $a \leq f \leq g \leq b$, and that, denoting by $\eta$ the unique increasing bijection from $[1, m]$ to $[f, g]$, one has $w_{2}=\eta \circ \bar{w} \circ \eta^{-1}$, and similarly for $y_{2}$ and $\bar{y}$.

Therefore, the permutations $w_{2}$ and $y_{2}$ can be read off from the graphs of $w$ and $y$ by "focusing" on the intersections of the columns $C_{i}, a \leq i \leq b$, and the lines $L_{j}, c \leq j \leq d$, see the example below. This is the explanation for the title of this section.

4.5. Example. In $S_{12}$ consider the permutations

$$
\begin{aligned}
& w=(6,7,2,9,12,10,11,4,1,5,3,8) ; \\
& y=(6,4,2,7,12,9,11,5,1,10,3,8) .
\end{aligned}
$$


Note that $y(i)=w(i)$ if $i<2$ or $i>10$ and that $y^{-1}(j)=w^{-1}(j)$ if $j<4$ or $j>10$.

It is helpful to represent each permutation $\tau \in S_{n}$ by its graph $G(\tau)$, that is, the set of points $(i, \tau(i))$ in $[1, n] \times[1, n]$. In the picture below, the graph of $w$ (resp. $y$ ) is represented by bullets and crosses (resp. bullets and stars).

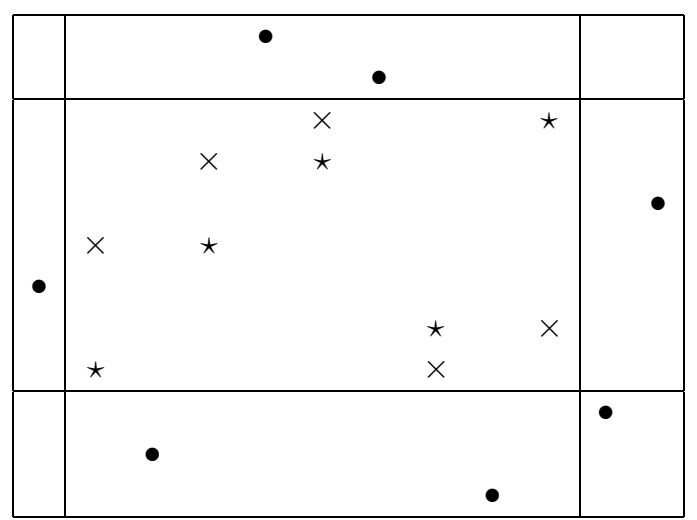

Then, the associated $\bar{w}$ and $\bar{y}$ in $S_{5}$ are respectively $(3,4,5,1,2)$ and $(1,3,4,2,5)$; they correspond to the pair associated to the polynomial $1+2 q$ in 1.4. Therefore, $P_{y, w}=1+2 q$.

\section{ACKNOWLEDGEMENTS}

This paper owes much to Fokko Du Cloux, who communicated to me several years ago his computer program "Coxeter" ([7]). It was on the ground of calculations made in the symmetric group $S_{8}$ that I was led to conjecture the theorem, as well as the form of the pair $y_{P}<w_{P}$. More recently, during a visit to the Institut Girard Desargues at Université Lyon I, discussions with Fokko du Cloux about this conjecture renewed my interest in the subject. It is a pleasure to thank him for sharing his program with me and for these discussions.

\section{REFERENCES}

1. H.H. Andersen, Schubert varieties and Demazure's character formula, Invent. Math. 79 (1985), 611-618. MR 86h:14042

2. A. Beilinson, J. Bernstein and P. Deligne, Faisceaux pervers, in "Analyse et topologie sur les espaces singuliers (I)", Astérisque 100, Soc. Math. France, 1982, pp. 3-171. MR 86g:32015

3. B.D. Boe, Kazhdan-Lusztig polynomials for hermitian symmetric spaces, Trans. Amer. Math. Soc. 309 (1988), 279-294. MR 89i:22024

4. M. Brion and P. Polo, Generic singularities of certain Schubert varieties, Math. Z. 231 (1999), 301-324.

5. V.V. Deodhar, Local Poincaré duality and nonsingularity of Schubert varieties, Comm. Algebra 13 (1985), 1379-1388. MR 86i:14015

6. V.V. Deodhar, A combinatorial setting for questions in Kazhdan-Lusztig theory, Geom. Dedicata 36 (1990), 95-119. MR 91h:20075

7. F. Du Cloux, program "Coxeter" (available at www.desargues.univ-lyon1.fr/home/ ducloux/coxeter.html).

8. M. Goresky and R. MacPherson, Intersection homology II, Invent. Math. 72 (1983), 77-129. MR 84i: 57012

9. R. Irving, The socle filtration of a Verma module, Ann. Sci. École Norm. Sup. 21 (1988), 47-65. MR 89h:17015 
10. B. Iversen, "Cohomology of sheaves", Springer-Verlag, Berlin Heidelberg 1986. MR 87 m:14013

11. D. Kazhdan and G. Lusztig, Representations of Coxeter groups and Hecke algebras, Invent. Math. 53 (1979), 165-184. MR 81j:20066

12. D. Kazhdan and G. Lusztig, Schubert varieties and Poincaré duality, in Proc. Symposia Pure Math. Vol. 36, Amer. Math. Soc., 1980, pp. 185-203. MR 84g:14054

13. A. Lascoux and M.P. Schützenberger, Polynômes de Kazhdan-Lusztig pour les grassmanniennes, in "Tableaux de Young et foncteurs de Schur en algèbre et géométrie", Astérisque 87-88, Soc. Math. France, 1981, pp. 249-266. MR 83i:14045

14. S. Ramanan and A. Ramanathan, Projective normality of flag varieties and Schubert varieties, Invent. Math. 79 (1985), 217-224. MR 86j:14051

15. C.S. Seshadri, Line bundles on Schubert varieties, in "Proceedings of the Bombay colloquium on vector bundles on algebraic varieties", 1984. MR 88i:14047

16. T.A. Springer, Quelques applications de la cohomologie d'intersection, Séminaire Bourbaki, Vol. 1981/1982, Astérisque, 92-93, Soc. Math. France, Paris, 1982, pp. 249-273. MR 85i:32016b

17. A. Zelevinsky, Small resolutions of singularities of Schubert varieties, Funct. Anal. Appl. 17 (1982), 142-144.

CNRS, UMR 7539, Institut Galilée, DéPartement de mathématiques, Université ParisNord, 93430 Villetaneuse, France

E-mail address: polo@math.univ-paris13.fr 